\title{
Quantum entanglement, spin-1/2 and the Stern-Gerlach experiment
}

\author{
G B Roston ${ }^{1}$, M Casas ${ }^{2}$, A Plastino ${ }^{3}$ and A R Plastino ${ }^{1,3}$ \\ ${ }^{1}$ Physics Department, University of Pretoria, Pretoria 0002, South Africa \\ 2 Departament de Física and IMEDEA-CSIC, Universitat de les Illes Balears, \\ E-07122 Palma de Mallorca, Spain \\ ${ }^{3}$ National University La Plata and CONICET, CC 727, (1900) La Plata, Argentina \\ E-mail: arplastino@maple.up.ac.za
}

Received 22 February 2005

Published 25 May 2005

Online at stacks.iop.org/EJP/26/657

\section{Abstract}

Basic aspects of quantum entanglement are illustrated in connection with the two-component wavefunction description of spin-1/2 particles. In particular, the time evolution of entanglement in the Stern-Gerlach experiment is analysed in detail.

\section{Introduction}

Quantum entanglement [1-9] has been the focus of intense research efforts in recent years [10-21]. There is a general consensus that quantum entanglement constitutes one of (if not the) most representative aspects of quantum mechanics. A state of a composite quantum system is called 'entangled' if it cannot be represented as a mixture of factorizable pure states. Otherwise, the state is called separable. The above definition is physically meaningful because entangled states (unlike separable states) cannot be prepared locally by acting on each subsystem individually [10]. Quantum entanglement can be regarded as a physical resource, which is associated with the peculiar non-classical correlations that are possible between separated quantum systems. Entanglement lies at the basis of important quantum information processes [11-13] such as quantum teleportation [14], superdense coding [15] and quantum computation [16, 17]. The experimental implementation of these processes may lead to a deep revolution in both the communication and computational technologies. Besides its possible practical applications, the study of quantum entanglement is of great importance in its own right. It constitutes an essential ingredient for a proper understanding of several fundamental aspects of physics, ranging from the quantum mechanical aspects of the second law of thermodynamics to the theory of quantum measurement, and the emergence of a 'classical world' from a quantum mechanical background.

In spite of being at the heart of quantum mechanics, a discussion of quantum entanglement is still lacking from standard quantum mechanics textbooks. Most of them do not even mention 
the word 'entanglement' in their subject indexes [22-27] (for recent notable exceptions see $[28,29])$. The aim of the present contribution is to illustrate some basic aspects of quantum entanglement in connection with a subject that is normally included in standard university courses and textbooks on quantum mechanics: the discussion of the kinematics and dynamics of spin-1/2 particles using two-component wavefunctions. Quantum entanglement arises from the tensor product structure of the Hilbert space describing composite quantum systems. This tensor product structure is also present when one deals with one system exhibiting various degrees of freedom. This case was considered, for instance, by de la Torre in [3]. An important example of a tensor product Hilbert space associated with a system with different degrees of freedom is provided by a particle with spin. Indeed, the study of spin usually constitutes the first encounter of quantum mechanics students with tensor product Hilbert spaces. Consequently, textbook chapters on the two-component wavefunctions formalism for spin- $1 / 2$ particles constitute appropriate places in which to incorporate an elementary discussion of quantum entanglement. Accordingly, we address the following aspects of entanglement: (i) the difference between separable and entangled states, (ii) a quantitative measure of entanglement, and (iii) an illustration of the relevance of entanglement for quantum mechanical measurement processes. This is done by recourse to a detailed analysis of the time evolution of entanglement during the Stern-Gerlach experiment [30-34].

\section{Spin-1/2 particles and entanglement}

\subsection{Two-component wavefunction}

Let us consider a pure state of a spin- $1 / 2$ particle described by the state vector

$$
|\Psi\rangle=\int \mathrm{d}^{3} r\left[f_{+}(\mathbf{r})|\mathbf{r}\rangle|+\rangle+f_{-}(\mathbf{r})|\mathbf{r}\rangle|-\rangle\right],
$$

where $f_{+}(\mathbf{r})$ and $f_{-}(\mathbf{r})$ are complex-valued functions of the position vector $\mathbf{r}$, and

$$
|+\rangle=\left(\begin{array}{l}
1 \\
0
\end{array}\right), \quad|-\rangle=\left(\begin{array}{l}
0 \\
1
\end{array}\right),
$$

are the eigenstates of the $z$-spin component operator $S_{z}$ with eigenvalues $+\hbar / 2$ and $-\hbar / 2$, respectively. The normalization of the state (1) is

$$
\langle\Psi \mid \Psi\rangle=\int \mathrm{d}^{3} r\left|f_{+}\right|^{2}+\int \mathrm{d}^{3} r\left|f_{-}\right|^{2}=1 .
$$

Even if the complete state (1) of the particle is pure, the spin degrees of freedom and the translational degrees of freedom are, when considered in their own right, described in general by mixed states. In order to determine the states characterizing separately the spin and the translational degrees of freedom, it is convenient to work with the density matrix associated with the pure state (1), which reads

$$
\begin{aligned}
& \hat{\rho}=|\Psi\rangle\langle\Psi| \\
& =\iint \mathrm{d}^{3} r \mathrm{~d}^{3} r^{\prime}\left[f_{+}(\mathbf{r})|\mathbf{r}\rangle|+\rangle+f_{-}(\mathbf{r})|\mathbf{r}\rangle|-\rangle\right]\left[f_{+}^{*}(\mathbf{r})\langle\mathbf{r}|\langle+|+f_{-}^{*}(\mathbf{r})\langle\mathbf{r}|\langle-|\right], \\
& \text { i.e., } \\
& \hat{\rho}=|+\rangle\left\langle+\left|\iint \mathrm{d}^{3} r \mathrm{~d}^{3} r^{\prime} f_{+}(\mathbf{r}) f_{+}^{*}\left(\mathbf{r}^{\prime}\right)\right| \mathbf{r}\right\rangle\left\langle\mathbf{r}^{\prime}|+|+\right\rangle\left\langle-\left|\iint \mathrm{d}^{3} r \mathrm{~d}^{3} r^{\prime} f_{+}(\mathbf{r}) f_{-}^{*}\left(\mathbf{r}^{\prime}\right)\right| \mathbf{r}\right\rangle\left\langle\mathbf{r}^{\prime}\right| \\
& +|-\rangle\left\langle+\left|\iint \mathrm{d}^{3} r \mathrm{~d}^{3} r^{\prime} f_{-}(\mathbf{r}) f_{+}^{*}\left(\mathbf{r}^{\prime}\right)\right| \mathbf{r}\right\rangle\left\langle\mathbf{r}^{\prime}\right| \\
& +|-\rangle\left\langle-\left|\iint \mathrm{d}^{3} r \mathrm{~d}^{3} r^{\prime} f_{-}(\mathbf{r}) f_{-}^{*}\left(\mathbf{r}^{\prime}\right)\right| \mathbf{r}\right\rangle\left\langle\mathbf{r}^{\prime}\right| .
\end{aligned}
$$


Now, the states characterizing 'individually' the spin and the position degrees of freedom are given, respectively, by the partial traces of $\hat{\rho}$ with respect to the spatial and spin variables. We proceed now to evaluate the partial trace of $\hat{\rho}$ over the position coordinates

$$
\hat{\rho}^{(S)}=\operatorname{Tr}_{\mathbf{r}}(\hat{\rho}) \text {. }
$$

If we previously define $|\mathbf{r},+\rangle \equiv|\mathbf{r}\rangle|+\rangle,\langle\mathbf{r},+|\equiv\langle\mathbf{r}|\langle+|,| \mathbf{r},-\rangle \equiv| \mathbf{r}\rangle|-\rangle$, and $\langle\mathbf{r},-| \equiv$ $\langle\mathbf{r}|\langle-|$, its four matrix elements read

$$
\begin{aligned}
& \hat{\rho}_{++}^{(S)}=\left\langle+\left|\hat{\rho}^{(S)}\right|+\right\rangle=\int \mathrm{d}^{3} r\langle\mathbf{r},+|\hat{\rho}| \mathbf{r},+\rangle \\
& \hat{\rho}_{+-}^{(S)}=\left\langle+\left|\hat{\rho}^{(S)}\right|-\right\rangle=\int \mathrm{d}^{3} r\langle\mathbf{r},+|\hat{\rho}| \mathbf{r},-\rangle \\
& \hat{\rho}_{-+}^{(S)}=\left\langle-\left|\hat{\rho}^{(S)}\right|+\right\rangle=\int \mathrm{d}^{3} r\langle\mathbf{r},-|\hat{\rho}| \mathbf{r},+\rangle \\
& \hat{\rho}_{--}^{(S)}=\left\langle-\left|\hat{\rho}^{(S)}\right|-\right\rangle=\int \mathrm{d}^{3} r\langle\mathbf{r},-|\hat{\rho}| \mathbf{r},-\rangle .
\end{aligned}
$$

It is clear that

$$
\begin{aligned}
\hat{\rho}_{++}^{(S)} & =\iiint \mathrm{d}^{3} r \mathrm{~d}^{3} r^{\prime} \mathrm{d}^{3} r^{\prime \prime}\left\langle\mathbf{r}^{\prime \prime} \mid \mathbf{r}\right\rangle\left\langle\mathbf{r}^{\prime} \mid \mathbf{r}^{\prime \prime}\right\rangle f_{+}(\mathbf{r}) f_{+}^{*}\left(\mathbf{r}^{\prime}\right) \\
& =\int \mathrm{d}^{3} r^{\prime \prime} f_{+}\left(\mathbf{r}^{\prime \prime}\right) f_{+}^{*}\left(\mathbf{r}^{\prime \prime}\right) \\
& =\int \mathrm{d}^{3} r\left|f_{+}(\mathbf{r})\right|^{2}
\end{aligned}
$$

In an analogous fashion,

$$
\begin{aligned}
\hat{\rho}_{+-}^{(S)} & =\int \mathrm{d}^{3} r f_{+}(\mathbf{r}) f_{-}^{*}(\mathbf{r}) \\
\hat{\rho}_{-+}^{(S)} & =\int \mathrm{d}^{3} r f_{-}(\mathbf{r}) f_{+}^{*}(\mathbf{r}) \\
\hat{\rho}_{--}^{(S)} & =\int \mathrm{d}^{3} r f_{-}(\mathbf{r}) f_{-}^{*}(\mathbf{r}) \\
& =\int \mathrm{d}^{3} r\left|f_{-}(\mathbf{r})\right|^{2} .
\end{aligned}
$$

Note that

$$
\begin{aligned}
\operatorname{Tr}\left[\hat{\rho}^{(S)}\right] & =\hat{\rho}_{++}^{(S)}+\hat{\rho}_{--}^{(S)} \\
& =\int \mathrm{d}^{3} r\left[\left|f_{+}(\mathbf{r})\right|^{2}+\left|f_{-}(\mathbf{r})\right|^{2}\right] \\
& =1
\end{aligned}
$$

and

$$
\hat{\rho}_{+-}^{(S)}=\left[\hat{\rho}_{-+}^{(S)}\right]^{*} .
$$

The marginal density matrix describing the spin variables can then be written as

$$
\begin{aligned}
\hat{\rho}^{(S)} & =\hat{\rho}_{++}^{(S)}|+\rangle\left\langle+\left|+\hat{\rho}_{+-}^{(S)}\right|+\right\rangle\left\langle-\left|+\hat{\rho}_{-+}^{(S)}\right|-\right\rangle\left\langle+\left|+\hat{\rho}_{--}^{(S)}\right|-\right\rangle\langle-| \\
& =\hat{\rho}_{++}^{(S)}|+\rangle\left\langle+\left|+\hat{\rho}_{+-}^{(S)}\right|+\right\rangle\left\langle-\left|+\left[\hat{\rho}_{+-}^{(S)}\right]^{*}\right|-\right\rangle\left\langle+\left|+\left(1-\hat{\rho}_{++}^{(S)}\right)\right|-\right\rangle\langle-| .
\end{aligned}
$$




\subsection{Evaluating entanglement}

Let $\left|\Psi_{A B}\right\rangle$ be a bipartite pure state and $\hat{\rho}_{A}=\operatorname{Tr}_{B}\left[\left|\Psi_{A B}\right\rangle\left\langle\Psi_{A B}\right|\right]$ and $\hat{\rho}_{B}=\operatorname{Tr}_{A}\left[\left|\Psi_{A B}\right\rangle\left\langle\Psi_{A B}\right|\right]$ the marginal density operators for, respectively, the subsystems $A$ and $B$. The amount of entanglement $E$ of the state $\left|\Psi_{A B}\right\rangle$ is given by the von Neumann marginal entropies [10]

$$
E=S\left(\hat{\rho}_{B}\right)=S\left(\hat{\rho}_{A}\right) \text {. }
$$

An essential property of entanglement is that the amount of entanglement does not change under local unitary operations. That is, the amount of entanglement does not change under the action of unitary operators of the form

$$
U=U_{A} \otimes U_{B},
$$

where $U_{A}$ and $U_{B}$ are unitary operators acting, respectively, upon subsystems $A$ and $B$. Let us now consider a Hamiltonian of the form

$$
H=H_{A} \otimes I_{B}+I_{A} \otimes H_{B},
$$

where $H_{A}$ and $H_{B}$ act, respectively, on subsystems $A$ and $B$, and $I_{A, B}$ are the identity operators associated with each subsystem's Hilbert space. The time evolution operator associated with the Hamiltonian (15) is a local unitary operator,

$$
U=\exp [-\mathrm{i} H t / \hbar]=\exp \left[-\mathrm{i} H_{A} t / \hbar\right] \otimes \exp \left[-\mathrm{i} H_{B} t / \hbar\right] .
$$

Consequently, the amount of entanglement does not change under the time evolution associated with the Hamiltonian (15). The time evolution operator associated with a Hamiltonian of the form

$$
H=H_{A} \otimes I_{B},
$$

which is

$$
U=\exp [-\mathrm{i} H t / \hbar]=\exp \left[-\mathrm{i} H_{A} t / \hbar\right] \otimes I_{B},
$$

is also local, and does not modify the amount of entanglement of the composite system. The same, obviously, happens with a Hamiltonian of the form $H=I_{A} \otimes H_{B}$.

The entanglement of the pure state $|\Psi\rangle$ is given by the von Neumann entropy of the marginal density matrix describing one of our two subsystems, say, that describing 'spin'

$$
E[|\Psi\rangle]=-\operatorname{Tr}\left[\hat{\rho}^{(S)} \ln \left(\hat{\rho}^{(S)}\right)\right]
$$

Let $\{|0\rangle,|1\rangle\}$ denote the eigenvectors of $\hat{\rho}^{(S)}$. Also, let its eigenvalues be $\left\{\lambda_{0}, \lambda_{1}\right\}$. With these quantities we can express $|\Psi\rangle$ according to its Schmidt decomposition [4]

$$
|\Psi\rangle=\int \mathrm{d}^{3} r\left[\sqrt{\lambda_{0}} f_{0}(\mathbf{r})|\mathbf{r}\rangle|0\rangle+\sqrt{\lambda_{1}} f_{1}(\mathbf{r})|\mathbf{r}\rangle|1\rangle\right] .
$$

The functions $f_{0}(\mathbf{r})$ and $f_{1}(\mathbf{r})$ are, in general, different from $f_{+}(\mathbf{r})$ and $f_{-}(\mathbf{r})$ that we used above. Of course,

$$
f_{0}(\mathbf{r})=\frac{1}{\sqrt{\lambda_{0}}}\langle\mathbf{r}, 0 \mid \Psi\rangle, \quad f_{1}(\mathbf{r})=\frac{1}{\sqrt{\lambda_{1}}}\langle\mathbf{r}, 1 \mid \Psi\rangle .
$$

In order to determine $f_{0}(\mathbf{r})$ and $f_{1}(\mathbf{r})$ one needs to

- build up $\hat{\rho}^{(S)}$,

- diagonalize it so as to find $\left\{\lambda_{0}, \lambda_{1}\right\},|0\rangle$, and $|1\rangle$,

- compute (21). 
The marginal density operator $\hat{\rho}^{(T)}\left(\mathbf{r}, \mathbf{r}^{\prime}\right)$,

$$
\begin{aligned}
\hat{\rho}^{(T)}\left(\mathbf{r}, \mathbf{r}^{\prime}\right) & =\operatorname{Tr}_{S}[|\Psi\rangle\langle\Psi|] \\
& =\left\langle\mathbf{r}, 0|\hat{\rho}| \mathbf{r}^{\prime}, 0\right\rangle+\left\langle\mathbf{r}, 1|\hat{\rho}| \mathbf{r}^{\prime}, 1\right\rangle \\
& =\lambda_{0} f_{0}(\mathbf{r}) f_{0}^{*}\left(\mathbf{r}^{\prime}\right)+\lambda_{1} f_{1}(\mathbf{r}) f_{1}^{*}\left(\mathbf{r}^{\prime}\right),
\end{aligned}
$$

describes the translational part of our 'composite' system.

Remember that if $\hat{A}$ is any Hermitian operator, $\{|i\rangle\}$ its eigenvectors, and $\left\{a_{i}\right\}$ its eigenvalues, then

$$
\hat{A}=\sum_{i} a_{i}|i\rangle\langle i|,
$$

and, for any analytical function $f(x)$,

$$
f(\hat{A})=\sum_{i} f\left(a_{i}\right)|i\rangle\langle i|,
$$

so that, in the case $f(x)=x \ln x$, we have

$$
\hat{A} \ln \hat{A}=\sum_{i} a_{i} \ln a_{i}|i\rangle\langle i|,
$$

and

$$
-\operatorname{Tr}\{\hat{A} \ln \hat{A}\}=-\sum_{i} a_{i} \ln a_{i} .
$$

Thus,

$$
\begin{aligned}
& E[|\Psi\rangle]=-\operatorname{Tr}\left[\hat{\rho}^{(S)} \ln \left(\hat{\rho}^{(S)}\right)\right]=-\sum_{i=0}^{1} \lambda_{i} \ln \lambda_{i} . \\
& \operatorname{det}\left(\hat{\rho}^{(S)}-\lambda I\right)=\operatorname{det}\left(\begin{array}{cc}
\rho_{++}^{(S)}-\lambda & \rho_{+-}^{(S)} \\
\rho_{-+}^{(S)} & \rho_{--}^{(S)}-\lambda
\end{array}\right)=0, \\
& \lambda_{1,2}=\frac{1}{2} \pm \frac{1}{2} \sqrt{1-4 \operatorname{det}\left(\hat{\rho}^{(S)}\right)} .
\end{aligned}
$$

\subsection{One-dimensional Gaussian wave packets}

We now consider states of a spin-1/2 particle (moving in one dimension) described by two Gaussian wave packets,

$$
\begin{aligned}
& f_{+}(x)=\alpha A_{+} \exp \left[\mathrm{i} k_{+}\left(x-x_{+}\right)-a_{+}^{2}\left(x-x_{+}\right)^{2} / 2\right], \\
& f_{-}(x)=\beta A_{-} \exp \left[\mathrm{i} k_{-}\left(x-x_{-}\right)-a_{-}^{2}\left(x-x_{-}\right)^{2} / 2\right],
\end{aligned}
$$

with

$$
A_{+}=\frac{1}{\pi^{1 / 4}} \sqrt{a_{+}}, \quad A_{-}=\frac{1}{\pi^{1 / 4}} \sqrt{a_{-}},
$$

where $a_{ \pm} \geqslant 0, k_{ \pm}$, and $x_{ \pm}$are real constants. The quantities $a_{ \pm}$are related to the widths of the wave packets through

$$
a_{+}=\frac{1}{\sigma_{+}}, \quad a_{-}=\frac{1}{\sigma_{-}} .
$$


The probability densities associated with the wave packets $f_{ \pm},\left|f_{+}(x)\right|^{2}$ and $\left|f_{-}(x)\right|^{2}$ are centred, respectively, at $x=x_{+}$and $x=x_{-}$. Note that in this case, the normalization of the state (1) implies that

$$
|\alpha|^{2}+|\beta|^{2}=1 .
$$

In what follows, we need the result

$$
\int_{-\infty}^{+\infty} \mathrm{d} x \exp \left[c_{1} x-c_{2} x^{2}\right]=\exp \left(\frac{c_{1}^{2}}{4 c_{2}}\right) \sqrt{\frac{\pi}{c_{2}}} .
$$

We have to evaluate the spin density matrix elements,

$$
\hat{\rho}_{L K}^{(S)}=\int_{-\infty}^{+\infty} \mathrm{d} x f_{L}(x) f_{K}^{*}(x) \quad L=+ \text { or }-\quad K=+ \text { or }-.
$$

One has

$$
\begin{aligned}
\hat{\rho}_{++}^{(S)} & =|\alpha|^{2} \\
\hat{\rho}_{--}^{(S)} & =|\beta|^{2} \\
\hat{\rho}_{+-}^{(S)} & =\left(\hat{\rho}_{-+}^{(S)}\right)^{*} \\
& =\alpha \beta^{*} v \exp (\xi) \exp (\mathrm{i} \theta) .
\end{aligned}
$$

where

$$
\begin{aligned}
& v=\frac{\sqrt{2 a_{+} a_{-}}}{\sqrt{a_{+}^{2}+a_{-}^{2}}} \\
& \xi=-\frac{\left(k_{+}-k_{-}\right)^{2}+a_{+}^{2} a_{-}^{2}\left(x_{+}-x_{-}\right)^{2}}{2\left(a_{+}^{2}+a_{-}^{2}\right)} \\
& \theta=\frac{\left(x_{-}-x_{+}\right)\left(a_{+}^{2} k_{-}+a_{-}^{2} k_{+}\right)}{\left(a_{+}^{2}+a_{-}^{2}\right)} .
\end{aligned}
$$

Now, from

$$
\operatorname{det}(\hat{\rho}-\lambda I)=\left|\begin{array}{cc}
|\alpha|^{2}-\lambda & \alpha \beta^{*} \nu \exp (\xi) \exp (\mathrm{i} \theta) \\
\beta \alpha^{*} \nu \exp (\xi) \exp (-\mathrm{i} \theta) & |\beta|^{2}-\lambda
\end{array}\right|=0
$$

one gets

$$
\lambda_{1,2}=\frac{1}{2} \pm \frac{1}{2} \sqrt{1-4|\alpha|^{2}|\beta|^{2}\left[1-v^{2} \exp (2 \xi)\right]} .
$$

For the case $|\alpha|^{2}=|\beta|^{2}=\frac{1}{2}$ we have

$$
\lambda_{0}=\frac{1}{2}+\frac{v}{2} \exp (\xi), \quad \lambda_{1}=\frac{1}{2}-\frac{v}{2} \exp (\xi)
$$

while the associated eigenvectors are seen to be

$$
\begin{aligned}
& |0\rangle=\frac{1}{\sqrt{2}}\left(\begin{array}{c}
\exp (\mathrm{i} \theta) \\
1
\end{array}\right), \\
& |1\rangle=\frac{1}{\sqrt{2}}\left(\begin{array}{c}
1 \\
-\exp (-\mathrm{i} \theta)
\end{array}\right),
\end{aligned}
$$




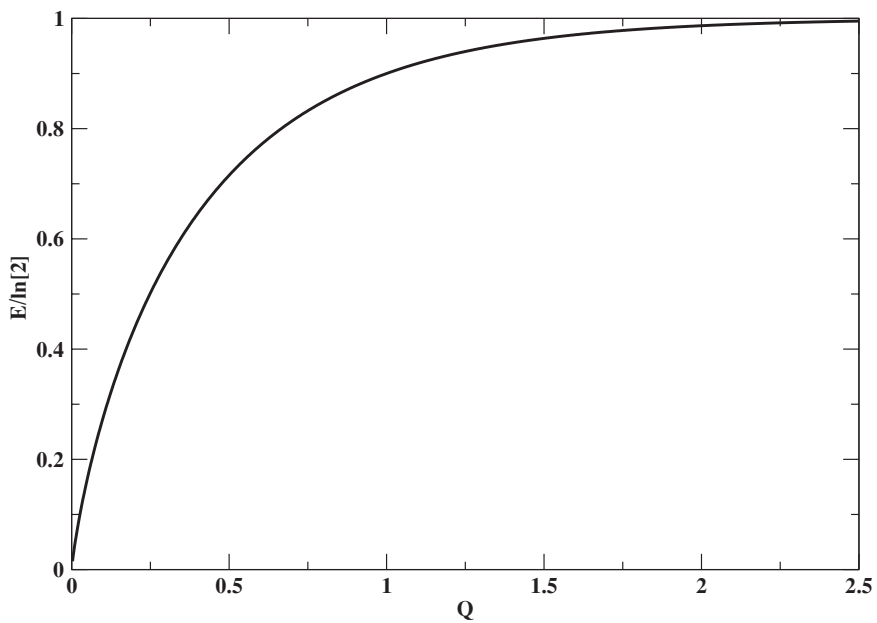

Figure 1. Entanglement $E[|\Psi\rangle] / \ln (2)$, as a function of $Q=\left(k_{+}-k_{-}\right)^{2} / 4 a^{2}\left(Q=\left(x_{+}-\right.\right.$ $\left.\left.x_{-}\right)^{2} a^{2} / 4\right)$, for a spin-1/2 particle's wavefunction with both components of Gaussian form, with $\alpha^{2}=\beta^{2}=0.5, a_{+}=a_{-}=a$, and $x_{+}=x_{-}\left(k_{+}=k_{-}\right)$. All depicted quantities are dimensionless.

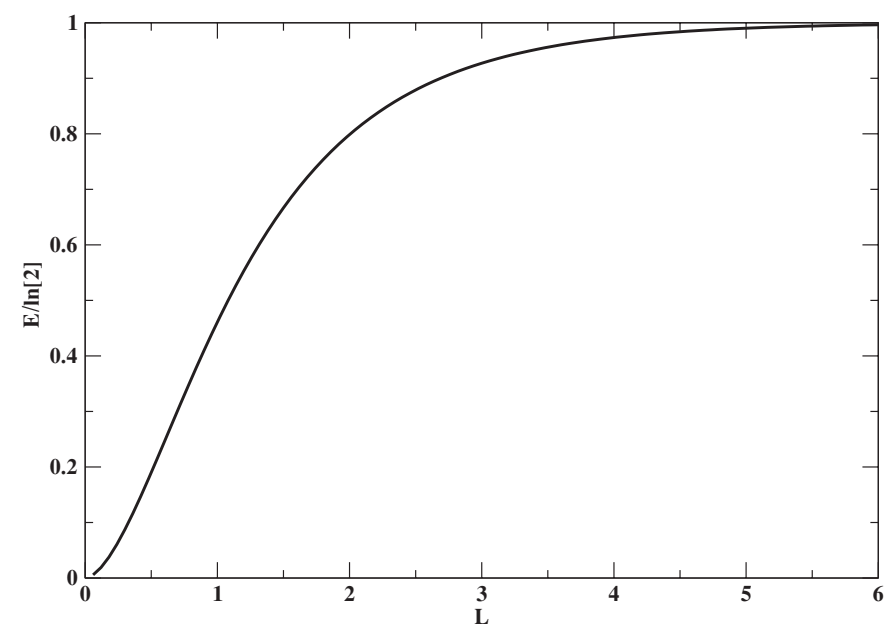

Figure 2. Entanglement $E[|\Psi\rangle] / \ln (2)$, as a function of $L=\ln \left(a_{+} / a_{-}\right)$, for a spin- $1 / 2$ particle's wavefunction with both components of Gaussian form, with $\alpha^{2}=\beta^{2}=0.5, x_{+}=x_{-}$, and $k_{+}=k_{-}$. All depicted quantities are dimensionless.

so that our entanglement $E$ turns out to be

$$
E[\Psi]=-\left[\frac{1}{2}+\frac{v}{2} \exp (\xi)\right] \ln \left[\frac{1}{2}+\frac{v}{2} \exp (\xi)\right]-\left[\frac{1}{2}-\frac{v}{2} \exp (\xi)\right] \ln \left[\frac{1}{2}-\frac{v}{2} \exp (\xi)\right] .
$$

Note that if $a_{+}=a_{-}, k_{+}=k_{-}$, and $x_{+}=x_{-}$, then $E[\Psi]=0$. On the other hand, if $x_{+}-x_{-} \rightarrow \infty$ or $k_{+}-k_{-} \rightarrow \infty$, then $E[\Psi]=\ln 2$. In figure 1 , the amount of entanglement $E[|\Psi\rangle] / \ln (2)$ is depicted as a function of $Q=\left(k_{+}-k_{-}\right)^{2} / 4 a^{2}\left(Q=\left(x_{+}-x_{-}\right)^{2} a^{2} / 4\right)$, taking $a_{+}=a_{-}=a$ and $x_{+}=x_{-}\left(k_{+}=k_{-}\right)$. In figure 2, $E[|\Psi\rangle] / \ln (2)$ is plotted against $L=\ln \left(a_{+} / a_{-}\right)$, taking $x_{+}=x_{-}$and $k_{+}=k_{-}$. 


\section{Time-dependent wavefunctions and entanglement}

\subsection{The free particle}

Now we consider a free, spin-1/2 particle. The Hamiltonian consists only of translational kinetic energy,

$$
H=\frac{P^{2}}{2 m}=-\frac{\hbar^{2}}{2 m}\left(\begin{array}{cc}
\partial^{2} / \partial x^{2} & 0 \\
0 & \partial^{2} / \partial x^{2}
\end{array}\right) .
$$

The well-known Gaussian wave packet solutions corresponding to a quantum free particle (with no spin) of mass $m$ moving in one dimension is

$$
\phi(x, t)=A(t) \exp \left[\frac{\frac{-a^{2}\left(x-x_{0}\right)^{2}}{2}+\mathrm{i} k\left[\left(x-x_{0}\right)-\frac{\hbar k t}{2 m}\right]}{1+\mathrm{i} \hbar a^{2} t / m}\right],
$$

where

$$
A(t)=\sqrt{\frac{(\sqrt{\pi})^{-1} a}{1+\mathrm{i}\left(\hbar a^{2} t / m\right)}} .
$$

The square modulus of this wavefunction, describing the (time-dependent) probability density for finding the particle at different positions, is given by

$$
|\phi(x, t)|^{2}=\frac{1}{\sqrt{2 \pi} \sigma(t)} \exp \left[-\frac{\left[\left(x-x_{0}\right)-\gamma t\right]^{2}}{2 \sigma(t)^{2}}\right],
$$

where

$$
\sigma(t)=\sqrt{\frac{1+\hbar^{2} a^{4} t^{2} m^{-2}}{2 a^{2}}}
$$

and

$$
\gamma=\hbar k / m
$$

Note that the wave packet (45) is normalized,

$$
\int|\phi(x, t)|^{2} \mathrm{~d} x=1
$$

A particular family of time-dependent solutions for a free spin-1/2 particle moving in one dimension is obtained by adopting Gaussian solutions of the form (45) for the two components $f_{ \pm}$of the particle's wavefunction,

$$
f_{+}(x, t)=\alpha A_{+}(t) \exp \left[\frac{-a_{+}^{2}\left(x-x_{+}\right)^{2} / 2+\mathrm{i} k_{+}\left[\left(x-x_{+}\right)-\frac{\hbar k_{+} t}{2 m}\right]}{1+\mathrm{i} \hbar m^{-1} a_{+}^{2} t}\right],
$$

and

$$
f_{-}(x, t)=\beta A_{-}(t) \exp \left[\frac{-a_{-}^{2}\left(x-x_{-}\right)^{2} / 2+\mathrm{i} k_{-}\left[\left(x-x_{-}\right)-\frac{\hbar k_{-} t}{2 m}\right]}{1+\mathrm{i} \hbar m^{-1} a_{-}^{2} t}\right],
$$

so that

$$
f_{+}(x, t) f_{+}^{*}(x, t)=\alpha^{2}\left|A_{+}(t)\right|^{2} \exp \left[-\frac{\left[\left(x-x_{+}\right)-\gamma_{+} t\right]^{2}}{2 \sigma_{+}^{2}(t)}\right]
$$

with

$$
\sigma_{+}(t)=\sqrt{\frac{1+\hbar^{2} a_{+}^{4} t^{2} m^{-2}}{2 a_{+}^{2}}}
$$


Analogously,

$$
f_{-}(x, t) f_{-}^{*}(x, t)=\beta^{2}\left|A_{-}(t)\right|^{2} \exp \left[-\frac{\left[\left(x-x_{-}\right)-\gamma_{-} t\right]^{2}}{2 \sigma_{-}^{2}(t)}\right],
$$

with

$$
\sigma_{-}(t)=\sqrt{\frac{1+\hbar^{2} a_{-}^{4} t^{2} m^{-2}}{2 a_{-}^{2}}}
$$

so that

$$
f_{+}(x, t) f_{-}^{*}(x, t)=\alpha \beta^{*} A_{+}(t)\left[A_{-}(t)\right]^{*} \exp \left[\frac{K_{+}}{1+\mathrm{i} \hbar m^{-1} a_{+}^{2} t}\right] \exp \left[\frac{K_{-}^{*}}{1-\mathrm{i} \hbar m^{-1} a_{-}^{2} t}\right],
$$

where

$$
\begin{aligned}
& K_{+}=-a_{+}^{2}\left(x-x_{+}\right)^{2} / 2+\mathrm{i} k_{+}\left[\left(x-x_{+}\right)-\frac{\hbar k_{+} t}{2 m}\right], \\
& K_{-}^{*}=-a_{-}^{2}\left(x-x_{-}\right)^{2} / 2-\mathrm{i} k_{-}\left[\left(x-x_{-}\right)-\frac{\hbar k_{-} t}{2 m}\right] .
\end{aligned}
$$

We calculate now $\hat{\rho}^{(S)}$ following (9) and using (34), (53) and (55):

$$
\begin{aligned}
& \hat{\rho}_{++}^{(S)}=\int_{-\infty}^{+\infty} \mathrm{d} x f_{+}(x, t) f_{+}^{*}(x, t)=|\alpha|^{2}, \\
& \hat{\rho}_{--}^{(S)}=\int_{-\infty}^{+\infty} \mathrm{d} x f_{-}(x, t) f_{-}^{*}(x, t)=|\beta|^{2} .
\end{aligned}
$$

For

$$
\hat{\rho}_{+-}^{(S)}=\int_{-\infty}^{+\infty} \mathrm{d} x f_{+}(x, t) f_{-}^{*}(x, t)
$$

instead, a little additional algebra is needed so as to get the final result. After using (57),

$\hat{\rho}_{+-}^{(S)}=\alpha \beta^{*} A_{+}(t)\left[A_{-}(t)\right]^{*} \exp (\tau) \int_{-\infty}^{+\infty} \mathrm{d} x \exp \left[-\frac{\left(a_{+}^{2}+a_{-}^{2}\right)}{2 K_{I}} x^{2}+\frac{\delta}{K_{I}} x\right]$,

with

$$
K_{I}=1+\mathrm{i} \hbar t m^{-1}\left(a_{+}^{2}-a_{-}^{2}\right)+\hbar^{2} a_{+}^{2} a_{-}^{2} t^{2} m^{-2}
$$

$$
\begin{aligned}
\tau=\frac{1}{K_{I}}\left[\begin{array}{r}
- \\
\frac{a_{+}^{2} x_{+}^{2}}{2}-
\end{array}\right. & \frac{a_{-}^{2} x_{-}^{2}}{2}+\left(-\mathrm{i} k_{+}-\gamma_{+} t a_{-}^{2}\right)\left(x_{+}+\frac{\gamma_{+} t}{2}\right) \\
& \left.+\left(\mathrm{i} k_{-}-\gamma_{-} t a_{+}^{2}\right)\left(x_{-}+\frac{\gamma_{-} t}{2}\right)+\frac{1}{2} \frac{\mathrm{i} \hbar}{m} t\left(a_{+}^{2} a_{-}^{2}\right)\left(x_{+}^{2}-x_{-}^{2}\right)\right],
\end{aligned}
$$

and

$\delta=a_{+}^{2} x_{+}+a_{-}^{2} x_{-}+\mathrm{i}\left(k_{+}-k_{-}\right)+\mathrm{i} \hbar m^{-1} t a_{+}^{2} a_{-}^{2}\left(x_{-}-x_{+}\right)+t\left(\gamma_{-} a_{+}^{2}+\gamma_{+} a_{-}^{2}\right)$,

using (34) we are led to

$$
\hat{\rho}_{+-}^{(S)}=\alpha \beta^{*} \sqrt{\frac{2 a_{+} a_{-}}{\left(a_{+}^{2}+a_{-}^{2}\right)}} \exp (\tau) \exp \left[\frac{\delta^{2}}{2\left(a_{+}^{2}+a_{-}^{2}\right) K_{I}}\right] .
$$

Rearranging and regrouping things we can recast the above now in the fashion

$$
\hat{\rho}_{+-}^{(S)}=\alpha \beta^{*} \nu \exp (\xi) \exp (\mathrm{i} \theta)
$$


where the quantities $v, \xi$ and $\theta$ are given (in terms of $a_{ \pm}, x_{ \pm}$and $k_{ \pm}$) by equations (37). Using matrix notation, we find then the desired result for $\hat{\rho}^{(S)}$ as follows,

$$
\hat{\rho}^{(S)}=\left(\begin{array}{cc}
|\alpha|^{2} & \alpha \beta^{*} \nu \exp (\xi) \exp (\mathrm{i} \theta) \\
\beta \alpha^{*} \nu \exp (\xi) \exp (-\mathrm{i} \theta) & |\beta|^{2}
\end{array}\right),
$$

whose eigenvalues are

$$
\lambda_{1,2}=\frac{1}{2} \pm \frac{1}{2} \sqrt{1-4|\alpha|^{2}|\beta|^{2}\left[1-v^{2} \exp (2 \xi)\right]} .
$$

Of course, once we have the eigenvalues we can straightforwardly evaluate $E[|\Psi\rangle]$ from (27),

$$
E[|\Psi\rangle]=-\lambda_{1} \ln \lambda_{1}-\lambda_{2} \ln \lambda_{2} .
$$

We see that the marginal spin density matrix (67) is time independent. In point of fact, the density matrix (67) coincides with the matrix (36), which was obtained from a wavefunction equal to (51)-(52) at $t=0$. The time independence of (67) implies that its eigenvalues and the entanglement measure $E[|\Psi\rangle]$ are time independent as well. The reason for this is that the free particle's Hamiltonian (44) is of the form (17), and the associated time evolution operator is 'local'.

\subsection{A spin-1/2 particle in a uniform magnetic field}

In this section, we consider the time dependence of entanglement with reference to a spin$1 / 2$ system with magnetic moment $\left(e \hbar / 2 m_{e} c\right)$ subjected to the action of an external uniform magnetic field $B$ applied in the $z$-direction. The Hamiltonian reads

$$
H=\frac{\mathbf{P}^{2}}{2 m}-\left(\frac{e B}{m_{e} c}\right) S_{z},
$$

so that, defining first the Larmor frequency

$$
\omega=\frac{|e| B}{m_{e} c}
$$

we can express the Hamiltonian in the fashion

$$
H=\frac{\mathbf{P}^{2}}{2 m}+\omega S_{z},
$$

so that the time evolution operator becomes

$$
U(t, 0)=\exp \left(\frac{-\mathrm{i} H t}{\hbar}\right)=\exp \left(\frac{-\mathrm{i} w S_{z} t}{\hbar}\right) \exp \left(-\frac{\mathrm{i} t}{\hbar} \frac{\mathbf{P}^{2}}{2 m}\right) .
$$

This is to be applied to the initial state

$$
\left|\Psi_{0}\right\rangle=\int \mathrm{d}^{3} r\left[f_{+}(\mathbf{r}, 0)|+\rangle|\mathbf{r}\rangle+f_{-}(\mathbf{r}, 0)|-\rangle|\mathbf{r}\rangle\right],
$$

and one obtains

$$
|\Psi(t)\rangle=\int \mathrm{d}^{3} r\left[\exp \left(-\frac{\mathrm{i} w t}{2}\right) f_{+}(\mathbf{r}, t)|+\rangle|\mathbf{r}\rangle+\exp \left(\frac{\mathrm{i} w t}{2}\right) f_{-}(\mathbf{r}, t)|-\rangle|\mathbf{r}\rangle\right] .
$$

Thus, the density operator for this system is represented by the matrix

$$
\hat{\rho}^{(S)}=\left(\begin{array}{cc}
\int\left|f_{+}\right|^{2} \mathrm{~d}^{3} r & \mathrm{e}^{-\mathrm{i} w t} \int f_{+} f_{-}^{*} \mathrm{~d}^{3} r \\
\mathrm{e}^{\mathrm{i} w t} \int f_{+}^{*} f_{-} \mathrm{d}^{3} r & \int\left|f_{-}\right|^{2} \mathrm{~d}^{3} r
\end{array}\right),
$$


whose eigenvalues are easily seen to be time independent, which entails that the entanglement inherits this time invariance as well. The Hamiltonian of a spin-1/2 particle in an homogeneous magnetic field constitutes an example of a Hamiltonian of the form (15). As we have already mentioned, the concomitant time evolution operator is 'local' (in the sense that it acts separately on the translational and on the spin degrees of freedom) and, consequently, preserves the amount of entanglement of the system.

\section{Entanglement in the Stern-Gerlach experiment}

The Stern-Gerlach experiment [30-38] is one of the most important experiments in the history of physics [30]. Due to its conceptual relevance, it continues to be the focus of research efforts [34, 39, 40], and plays a fundamental role in the teaching of quantum mechanics $[24,28]$. Within our present approach, the Stern-Gerlach experiment also provides a valuable opportunity for discussing the role of entanglement in quantum measurement processes [34]. In the Stern-Gerlach experiment a beam of particles moving parallel to the $x$-axis are passed through a non-uniform magnetic field $\mathbf{B}$ contained in the plane perpendicular to this axis (so that $B_{x}=0$ ) of the form

$$
\mathbf{B}(\mathbf{r})=0 \hat{\mathbf{i}}+b y \hat{\mathbf{j}}+\left(B_{0}-b z\right) \hat{\mathbf{k}} .
$$

This field has a constant component of intensity $B_{0}$ and a gradient of intensity $b$. The Schrödinger equation describing the system is

$$
H|\psi\rangle=\mathrm{i} \hbar \frac{\partial}{\partial t}|\psi\rangle,
$$

with the Hamiltonian being [32]

$$
H=\frac{\mathbf{P}^{2}}{2 m}+\frac{g \mu_{B}}{\hbar} \mathbf{S} \cdot \mathbf{B}(\mathbf{r}),
$$

with $\mu_{B}$ the Bohr magneton and $g$ the gyromagnetic ratio. Substituting (79) and (77) into (78) we obtain coupled Schrödinger equations for each spinor component [32]

$$
\begin{aligned}
& -\frac{\hbar^{2}}{2 m} \nabla^{2} \psi_{+}+\frac{g \mu_{B} b y}{2 \mathrm{i}} \psi_{-}+\frac{g \mu_{B}}{2}\left(B_{0}-b z\right) \psi_{+}=\mathrm{i} \hbar \frac{\partial \psi_{+}}{\partial t}, \\
& -\frac{\hbar^{2}}{2 m} \nabla^{2} \psi_{-}-\frac{g \mu_{B} b y}{2 \mathrm{i}} \psi_{+}-\frac{g \mu_{B}}{2}\left(B_{0}-b z\right) \psi_{-}=\mathrm{i} \hbar \frac{\partial \psi_{-}}{\partial t} .
\end{aligned}
$$

The spinor components are seen to be coupled through the $\mathbf{S}_{y}$ contribution. Now, the spin $\mathbf{S}$ exhibits the famous precession effect about the axis $\hat{\mathbf{B}}(\mathbf{r}) \sim \hat{\mathbf{k}}$, according to [32]

$$
\frac{\mathrm{d} \mathbf{S}}{\mathrm{d} t}=-\frac{\mu_{B} g}{\hbar} \mathbf{B}(\mathbf{r}) \times \mathbf{S},
$$

with frequency $\omega \sim B_{0} \mu_{B} g / \hbar$. Here $S_{z}$ is preserved, but the $S_{y}$ contribution oscillates rapidly. It is argued in [32] that this oscillation, after properly averaging over time, effectively decouples our two equations. One then effects first the transformation [32]

$$
\begin{aligned}
& \psi_{+}(\mathbf{r}, t)=\exp \left(\frac{-\mathrm{i} g \mu_{B} B_{0} t}{2 \hbar}\right) \bar{\psi}_{+}(\mathbf{r}, t), \\
& \psi_{-}(\mathbf{r}, t)=\exp \left(\frac{+\mathrm{i} g \mu_{B} B_{0} t}{2 \hbar}\right) \bar{\psi}_{-}(\mathbf{r}, t),
\end{aligned}
$$

and, as stated, averaging over a time period (i) long enough compared to the oscillation period for the coupling term, but (ii) short compared with those needed for the packet to appreciably 
move along its trajectory, the coupling term relating the new barred component is seen to vanish [32], so that (80) becomes

$$
\begin{aligned}
& -\frac{\hbar^{2}}{2 m} \nabla^{2} \bar{\psi}_{+}-\frac{g \mu_{B} b z}{2} \bar{\psi}_{+}=\mathrm{i} \hbar \frac{\partial \bar{\psi}_{+}}{\partial t}, \\
& -\frac{\hbar^{2}}{2 m} \nabla^{2} \bar{\psi}_{-}+\frac{g \mu_{B} b z}{2} \bar{\psi}_{-}=\mathrm{i} \hbar \frac{\partial \bar{\psi}_{-}}{\partial t},
\end{aligned}
$$

and we have ended up with two independent equations, one for each component, each of which has been endowed with its own effective Hamiltonian corresponding to a particle subject to a force whose direction is determined by the spin quantum number [32]. These are the equations we have to tackle now. Given two pertinent solutions $\bar{\psi}_{ \pm}(\mathbf{r}, t)$, the time evolution in the Stern-Gerlach would then be given in the fashion

$$
\psi_{ \pm}(\mathbf{r}, t)=\exp \left(\mp \frac{\mathrm{i} g \mu_{B} B_{0} t}{2 \hbar}\right) \bar{\psi}_{ \pm}(\mathbf{r}, t) .
$$

Going back to (83), solving either of the two equations poses the problem of solving the Schrödinger equation for a particle subject to the action of a constant applied force in the $z$-direction. This Schrödinger equation is separable. That is, the product of two onedimensional solutions for a free particle moving along the $x$ - and $y$-axes, respectively, times a one-dimensional solution for a particle moving along the $z$-axis under the influence of a constant force, provides a solution for our three-dimensional problem. Now, it has been shown in [41] that there is a general procedure for the construction, starting from a solution to the (one-dimensional) free particle's Schrödinger equation, of a time-dependent solution of the Schrödinger equation corresponding to a particle subject to a constant force. Consequently, we start with a factorized, time-dependent solution for a (three-dimensional) free particle,

$$
\phi(\mathbf{r}, t)=\phi(x, t) \phi(y, t) \phi(z, t),
$$

where each factor is a one-dimensional Gaussian wave packet (see equation (45)). Then, following the procedure explained in [41], we construct the associated solution for the case of a constant force in the $z$-direction,

$$
\bar{\psi}(\mathbf{r}, t)=\phi(x, t) \phi(y, t) \bar{\psi}(z, t),
$$

where (only the factor involving the coordinate $z$ is affected)

$$
\bar{\psi}(z, t)=\phi\left(z-z_{0}-v_{0} t-\frac{1}{2} a_{c} t^{2}, t\right) \mathrm{e}^{\mathrm{i} S(z, t)} .
$$

Here $z_{0}$ and $v_{0}$ are integration constants and $a_{c}$ is the (uniform) acceleration. In our case, we have

$$
a_{c \pm}= \pm \frac{g \mu_{B} b}{2 m}
$$

In general, one has for $S(z, t)[41]$

$$
S(z, t)=\frac{m}{\hbar}\left(v_{0} z+a_{c} z t-\frac{1}{2} a_{c} v_{0} t^{2}-\frac{1}{6} a_{c}^{2} t^{3}-\frac{1}{2} v_{0}^{2} t+\text { constant }\right) .
$$

Using now (84)-(89) we can express the solution for the Stern-Gerlach experiment in the fashion

$$
\psi_{ \pm}(z, t)=\sqrt{\frac{(2 \sqrt{\pi})^{-1} a_{ \pm}}{1+\mathrm{i}\left(\hbar a_{ \pm}^{2} t / m\right)}} \exp \left[\frac{\frac{-a_{ \pm}^{2}\left[z-z_{ \pm}(t)\right]^{2}}{2}+\mathrm{i} k_{ \pm}\left[\left(z-z_{ \pm}(t)\right)-\frac{\hbar k_{ \pm} t}{2 m}\right]}{1+\mathrm{i} \hbar a_{ \pm}^{2} t / m}\right] \exp \left[\mathrm{i} T_{ \pm}(z, t)\right]
$$


with

$$
z_{ \pm}(t)=z_{0}+v_{0} t+\frac{1}{2} a_{c \pm} t^{2}
$$

and

$$
T_{ \pm}(z, t)=S(z, t) \mp \frac{g \mu_{B} B_{0} t}{2 \hbar} .
$$

Note that each component $\psi_{ \pm}$is normalized to $1 / 2$, in such a way that the complete twocomponent wavefunction is normalized to unity,

$$
\int\left[\left|\psi_{+}\right|^{2}+\left|\psi_{-}\right|^{2}\right] \mathrm{d}^{3} r=1
$$

We have at this point, with (90), all that is needed so as to evaluate the density operator $\hat{\rho}^{(S)}$. We now assume that $a_{+}=a_{-}=a, k_{+}=k_{-}, z_{0}=0$, and $v_{0}=0$. We find, following the procedure of the preceding sections,

$$
\hat{\rho}^{(S)}(t)=\left(\begin{array}{cc}
\frac{1}{2} & \int \psi_{+} \psi_{-}^{*} \mathrm{~d}^{3} r \\
\int \psi_{+}^{*} \psi_{-} \mathrm{d}^{3} r & \frac{1}{2}
\end{array}\right),
$$

the pertinent corresponding eigenvalues reading

$$
\lambda_{1,2}=\frac{1}{2} \pm\left|\int \psi_{+} \psi_{-}^{*} \mathrm{~d}^{3} r\right|
$$

where

$$
\left|\int \psi_{+} \psi_{-}^{*} \mathrm{~d}^{3} r\right|=\frac{1}{2} \exp (\eta)
$$

with

$$
\eta=\left(\frac{g \mu_{B} b}{2 m}\right)^{2} t^{2}\left[\frac{-4 m^{2} / \hbar^{2}-5 t^{2} a^{4}-a^{8} t^{4} \hbar^{2} / m^{2}}{4 a^{2}\left(1+\hbar^{2} a^{4} t^{2} / m^{2}\right)}\right] .
$$

Finally, by recourse to (27) we can evaluate the entanglement $E[|\Psi\rangle](t)$ in the present setting. We only have to insert the eigenvalues (95), computed by recourse to (96)-(97), into

$$
E[|\Psi\rangle](t)=-\lambda_{1} \ln \lambda_{1}-\lambda_{2} \ln \lambda_{2} .
$$

The evolution of the entanglement between the translational and the spin degrees of freedom in the Stern-Gerlach experiment is illustrated in figure 3. In this figure $E / \ln (2)$ is plotted against $t^{\prime}=\hbar a^{2} t / m$, for various values of the dimensionless quantity $\kappa=\frac{1}{a}\left[\frac{m^{2} a_{c}}{2 \hbar^{2}}\right]^{1 / 3}, \kappa=1$ (continuous line), $\kappa=0.5$ (dotted line), $\kappa=0.3$ (dashed line), and $\kappa=0.2$ (dot-dashed line).

We have also considered the contextuality measure $I$ discussed by de la Torre in [3]. The measure $I$ was inspired by Schrödinger's formulation of the uncertainty principle, given by [3]

$$
(\Delta \hat{A})^{2}(\Delta \hat{B})^{2} \geqslant\left(\frac{1}{2 \mathrm{i}}\langle[\hat{A}, \hat{B}]\rangle\right)^{2}+\left(\frac{1}{2}\langle\{\hat{A}, \hat{B}\}\rangle-\langle\hat{A}\rangle\langle\hat{B}\rangle\right)^{2},
$$

where an anticommuter appears in the second term of the right-hand side. This second term is precisely $I$, i.e.,

$$
I=\left[\frac{1}{2}\langle\{A, B\}\rangle-\langle A\rangle\langle B\rangle\right]^{2}:\{A, B\}=A B+B A .
$$

Consider the case when we have a composite quantum system consisting of two subsystems, and the observable $A$ refers to one of these subsystems, while observable $B$ refers to the other subsystem. It is clear that, under these circumstances, the quantity $I$ vanishes for all factorizable pure states. In contrast, it does not vanish for some entangled pure states. The same situation occurs when observables $A$ and $B$ refer to different degrees of freedom of a 


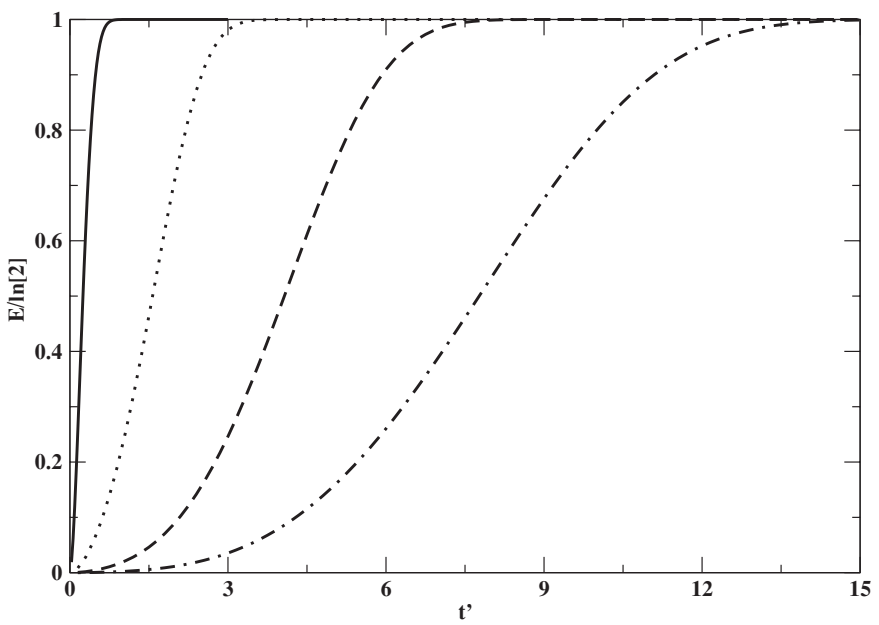

Figure 3. Time dependence of the entanglement for the Stern-Gerlach experiment. $E / \ln (2)$ is plotted against $t^{\prime}=\hbar a^{2} t / m$, for various values of the dimensionless quantity $\kappa=\frac{1}{a}\left[\frac{m^{2} a_{c}}{2 \hbar^{2}}\right]^{1 / 3}$ : $\kappa=1$ (continuous line), $\kappa=0.5$ (dotted line), $\kappa=0.3$ (dashed line), and $\kappa=0.2$ (dot-dashed line). All depicted quantities are dimensionless.

given system leading to a Hilbert space with a tensor product structure. This is the case when we have the translational and the spin degrees of freedom. Here we take $A=z$ and $B=S_{z}$, so that

$$
I=\left[\left\langle z S_{z}\right\rangle-\langle z\rangle\left\langle S_{z}\right\rangle\right]^{2}
$$

Evaluating now $I$ on the wavefunction (90) we find

$$
I=\frac{\hbar^{2}}{16}\left(z_{+}-z_{-}\right)^{2} .
$$

Therefore, the time dependence of $I$ in the Stern-Gerlach experiment is given by

$$
I=\frac{\hbar^{2}}{16} \frac{g^{2} \mu_{B}^{2} b^{2}}{4 m^{2}} t^{4} .
$$

We see that, as occurs with the entanglement measure $E$, the measure $I$ also increases monotonically with time during the Stern-Gerlach experiment.

\section{Conclusions}

In the present contribution, we have considered basic aspects of quantum entanglement in connection with the two-component wavefunction description of spin- $1 / 2$ particles. Spin- $1 / 2$ systems were used to illustrate several important concepts related to quantum entanglement: (i) the distinction between separable and entangled states, (ii) a quantitative measure of entanglement, and (iii) the entanglement preserving property of local unitary operations. This last aspect of entanglement was illustrated with two basic systems: a free, spin- $1 / 2$ particle and a spin-1/2 particle in a uniform magnetic field. Finally, we studied the time evolution of entanglement in the Stern-Gerlach experiment. We derived an expression for the amount of entanglement between the spin and the translational degrees of freedom as a function of time, and showed that it increases monotonically during the Stern-Gerlach experiment. We also 
computed, as a function of time, the measure $I$ introduced by de la Torre in [3]. This measure also increases monotonically.

The main message we wanted to convey in the present contribution is that the twocomponent wavefunction formalism offers interesting opportunities for the discussion of important ideas related to quantum entanglement, using ingredients that are usually included in standard courses in quantum mechanics. Of course, this is not the only scenario in terms of which entanglement can be illustrated in a quantum mechanics course. The standard case of a composite system constituted by two subsystems endowed with two-dimensional Hilbert spaces (that is, two qubits) can also be profitably discussed [1]. The discussion presented here, concerning the two-component wave formalism, should be studied in addition to two-qubit systems. The analysis of entanglement in relation to the two-component wave formalism is particularly interesting because it allows for the discussion of entanglement in the SternGerlach experiment, which constitutes the paradigmatic example of a quantum mechanical measurement process.

\section{Acknowledgments}

This work was partially supported by the MCyT-Feder grant no BMF 2002-03241, by the Government of the Balearic Islands (Spain), and by CONICET (Argentine Agency).

\section{References}

[1] Walck S N and Hansell N C 2001 Characterization and visualization of the state and entanglement of two spins Eur. J. Phys. 22 343-50

[2] Tommasini P, Timmermans E and Toledo Piza A F R 1998 The hydrogen atom as an entangled electron-proton system Am. J. Phys. 66 881-6

[3] de la Torre A C 1994 Contextuality in quantum systems Am. J. Phys. 62 808-12

[4] Ekert A and Knight P L 1995 Entangled quantum systems and the Schmidt decomposition Am. J. Phys. 63 415-23

[5] de la Torre A C and Dotson A C 1996 An entangled opinion on the interpretation of quantum mechanics Am.J. Phys. 64174

[6] Aravind P K 1996 Geometry of the Schmidt decomposition and Hardy's theorem Am. J. Phys. 64 1143-50

[7] Dehlinger D and Mitchell M W 2002 Entangled photons, nonlocality, and Bell inequalities in the undergraduate laboratory Am. J. Phys. 70 903-10

[8] Dehlinger D and Mitchell M W 2002 Entangled photon apparatus for the undergraduate laboratory Am. J. Phys. 70 898-902

[9] Paty M 1999 Are quantum systems physical objects with physical properties? Eur. J. Phys. 20 373-88

[10] Lo H-K, Popescu S and Spiller T (ed) 1998 Introduction to Quantum Computation and Information (River Edge: World Scientific)

[11] Williams C P and Clearwater S H 1997 Explorations in Quantum Computing (New York: Springer)

[12] Williams C P (ed) 1998 Quantum Computing and Quantum Communications (Berlin: Springer)

[13] Bouwmeester D, Ekert A and Zeilinger A (ed) 1998 The Physics of Quantum Information (Berlin: Springer)

[14] Bennett C H, Brassard G, Crepeau C, Jozsa R, Peres A and Wootters W K 1993 Teleporting an unknown quantum state via dual classical and Einstein-Podolsky-Rosen channels Phys. Rev. Lett. 70 1895-9

[15] Bennett C H and Wiesner S J 1993 Communication via one- and two-particle operators on Einstein-PodolskyRosen states Phys. Rev. Lett. $692881-4$

[16] Ekert A and Jozsa R 1996 Quantum computation and Shor's factoring algorithm Rev. Mod. Phys. $68733-53$

[17] Berman G P, Doolen G D, Mainieri R and Tsifrinovich V I 1998 Introduction to Quantum Computers (Singapore: World Scientific)

[18] Galindo A and Martin-Delgado M A 2002 Information and computation: classical and quantum aspects Rev. Mod. Phys. 74 347-423

[19] Batle J, Casas M, Plastino A R and Plastino A 2002 Entanglement, mixedness and q-entropies Phys. Lett. A $296251-7$

[20] Batle J, Casas M, Plastino A and Plastino A R 2005 Maximally entangled mixed states and conditional entropies Phys. Rev. A 71024301

[21] Jacak L 2000 Semiconductor quantum dots-towards a new generation of semiconductor devices Eur. J. Phys. 21 487-97 
[22] Griffiths D J 1995 Introduction to Quantum Mechanics (Englewood Cliffs, NJ: Prentice-Hall)

[23] Galindo A and Pascual P 1990 Quantum Mechanics (New York: Springer)

[24] Sakurai J J 1985 Modern Quantum Mechanics (New York: Addison-Wesley)

[25] Cohen-Tannoudji C, Diu B and Laloe F 1977 Quantum Mechanics (New York: Wiley)

[26] Park D 1974 Introduction to The Quantum Theory (New York: McGraw-Hill)

[27] Merzsbacher E 1970 Quantum Mechanics 2nd edn (New York: Wiley)

[28] Isham C J 2001 Lectures on Quantum Theory: Mathematical and Structural Foundations (London: Imperial College Press)

[29] Peres A 1993 Quantum Theory: Concepts and Methods (Dordrecht: Kluwer)

[30] Friedrich D and Herschbach D 2003 Stern and Gerlach: how a bad cigar helped reorient atomic physics Phys. Today 56 53-9

[31] Patil S H 1998 Quantum mechanical description of the Stern-Gerlach experiment Eur. J. Phys. 19 25-30

[32] Platt D 1992 A modern analysis of the Stern-Gerlach experiment Am. J. Phys. 60 306-8

[33] Mackintosh A R 1983 The Stern-Gerlach experiment, electron spin and intermediate quantum mechanics Eur. J. Phys. 4 97-106

[34] Reinisch G 1999 Stern-Gerlach experiment as the pioneer-and probably the simplest-quantum entanglement test? Phys. Lett. A 259 427-30

[35] Hannout M, Hoyt S, Kryowonos A and Widom A 1998 Quantum measurement theory and the Stern-Gerlach experiment Am. J. Phys. 66 377-9

[36] Schönhammer K 2000 Stern-Gerlach measurements with arbitrary spin: quantum combs Am. J. Phys. 68 49-55

[37] Batelaan H 2002 Electrons, Stern-Gerlach magnets, and quantum mechanical propagation Am. J. Phys. 70 325-31

[38] Porter J, Pettifer R F and Leadley D R 2003 Direct demonstration of the transverse Stern-Gerlach effect Am. J. Phys. 71 1103-8

[39] Banerjee S and Ghosh R 2000 Quantum theory of a Stern-Gerlach system with a linearly dissipative environment Phys. Rev. A 62042105

[40] Cruz-Barrios S and Gomez-Camacho J 2001 Semiclassical description of Stern-Gerlach experiments Phys. Rev. A 63012101

[41] Vandegrift G 2000 Accelerating wave packet solution to Schrödinger's equation Am. J. Phys. 68 576-7 